\title{
Multiple new-particle growth pathways observed at the US DOE South- ern Great Plains field site
}

\author{
Anna L. Hodshire et al. \\ Correspondence to: Anna L. Hodshire (hodshire@ rams.colostate.edu)
}

The copyright of individual parts of the supplement might differ from the CC-BY 3.0 licence. 


\section{Supplementary Information}

\section{S1 Growth Rate Methods}

Three methods were employed for determining a range of possible growth rates from the SMPS data. The first method, referred to here as the leading-edge method, is adapted from Lehtipalo et al. (2014) and finds the time at which the binned aerosol distribution between 10-20 nm reaches one-half of its maximum concentration for each bin. A linear fit between the bins' median diameter and the associated times determines the growth rate. The second method, referred to here as the $\mathrm{D}_{\mathrm{p}}$-mode method, tracks the change in diameter of the maximum concentration of the aerosol size distribution between 10-20 nm; a linear fit between peak diameters and the associated times determines the growth rate. When plotted against the size distribution (Figures S1-S3), it is seen that the leading-edge and $\mathrm{D}_{\mathrm{p}}$ mode methods do not tend to track the growing size distribution for every days. For this reason, we have included a third method, which we call the visual method, in which we have made a linear growth-rate estimate between 10-20 $\mathrm{nm}$ for each day based upon visual inspection of the size distribution.

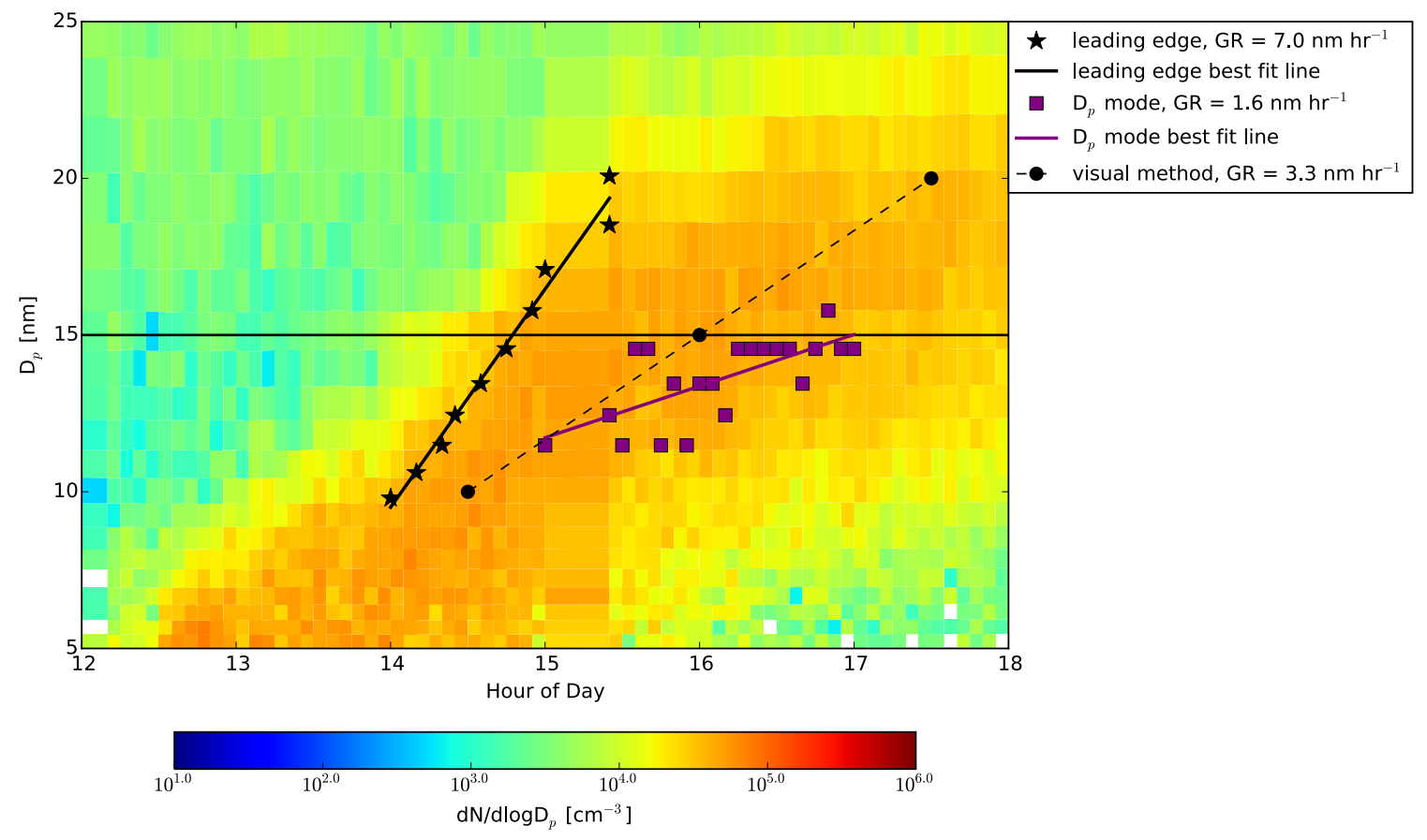

Figure S1. The results of the three growth rate calculations for April 19, 2013. The x-axis represents CDT time. The line at $15 \mathrm{~nm} \mathrm{D}_{\mathrm{p}}$ is to guide the eye. 


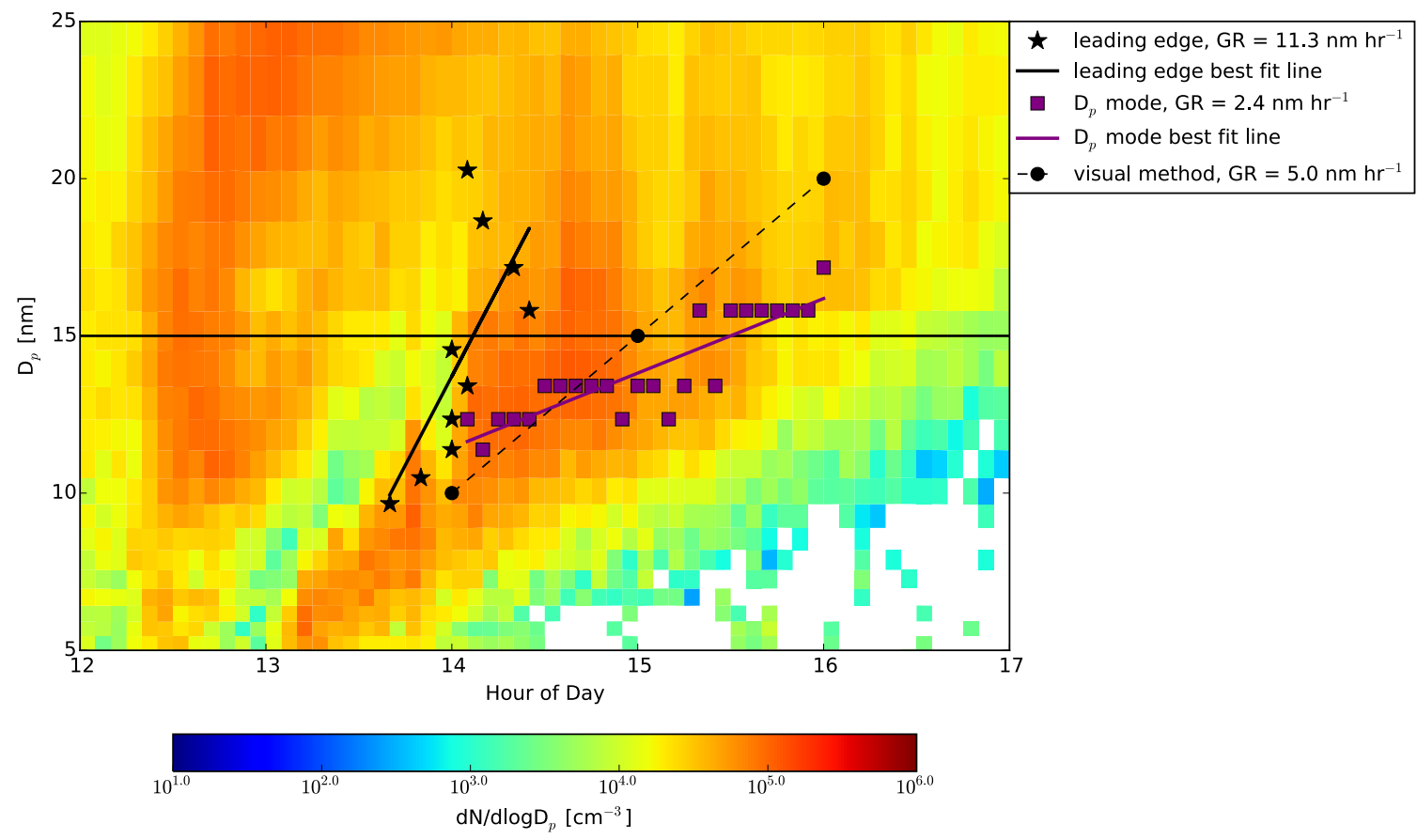

Figure S2. The results of the three growth rate calculations for May 9, 2013. The $\mathrm{x}$-axis represents CDT time. The line at $15 \mathrm{~nm} \mathrm{D}_{\mathrm{p}}$ is to guide the eye. 


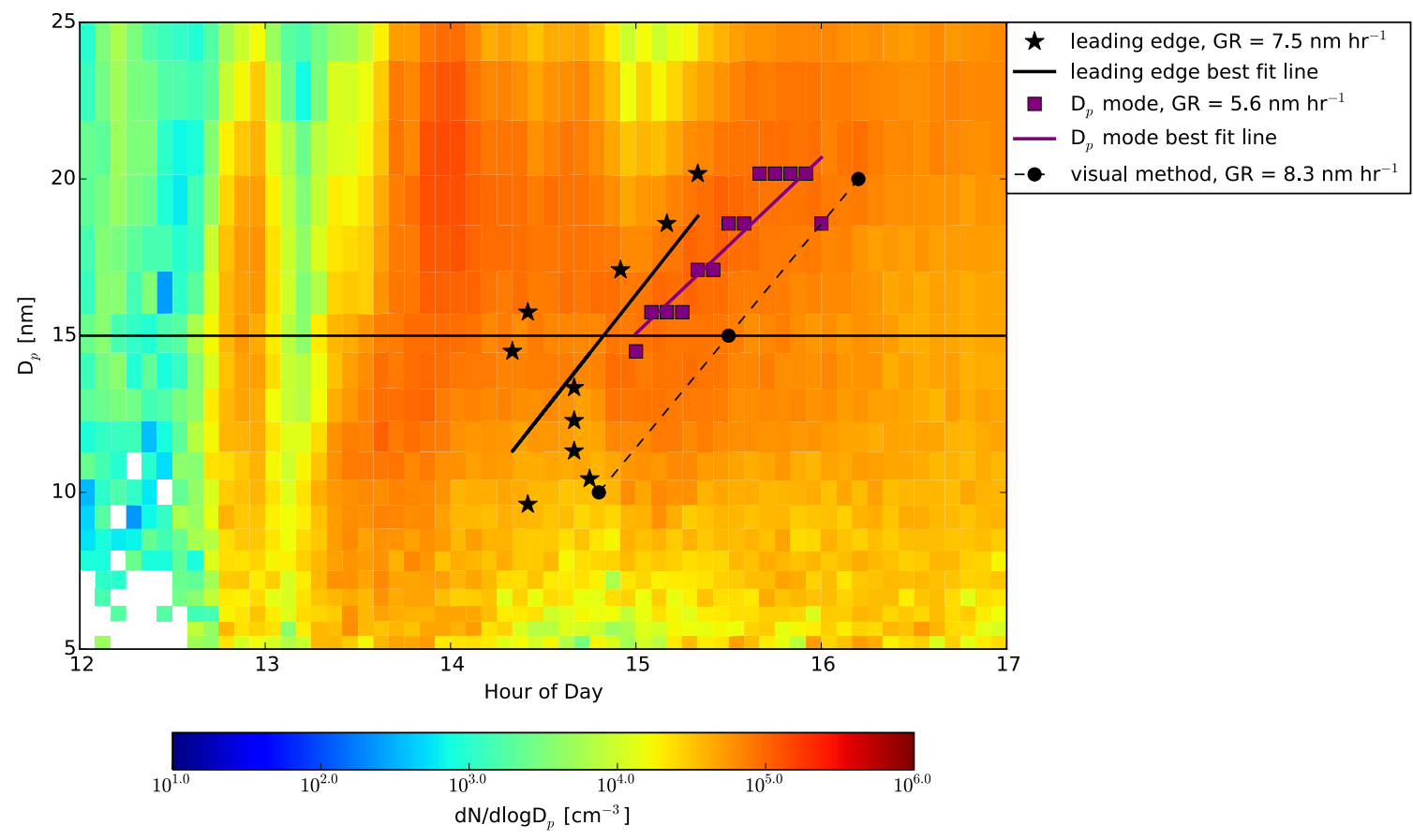

Figure S3. The results of the three growth rate calculations for May 11, 2013. The x-axis represents CDT time. The line at $15 \mathrm{~nm} \mathrm{D}_{\mathrm{p}}$ is to guide the eye. 
S2 MABNAG-predicted Ion Dissociations

Table S1. Breakdown of the percent dissociations of $\mathrm{H}_{2} \mathrm{SO}_{4}$ to $\mathrm{HSO}_{4}{ }^{-1}$ to $\mathrm{HSO}_{4}{ }^{-1}$ and $\mathrm{SO}_{4}{ }^{-2}$; the organic acid (malonic or oxalic) to its first and second dissociation products; $\mathrm{NH}_{3}$ to $\mathrm{NH}_{4}^{+}$; and the amine (DMA or TMA) to its dissociation product for the model output of April 19, 2013. In MABNAG, sulfuric acid is assumed to dissociate completely.

\begin{tabular}{|c|c|c|c|c|c|c|c|c|c|}
\hline Case & $\mathrm{HSO}_{4}^{-1}$ & $\mathrm{SO}_{4}^{-2}$ & $\begin{array}{l}\text { Org. } \\
\text { Acid }\end{array}$ & $\begin{array}{l}\text { Org. } \\
\text { Acid }^{-1}\end{array}$ & $\begin{array}{l}\text { Org. } \\
\text { Acid }^{-2}\end{array}$ & $\mathrm{NH}_{3}$ & $\mathrm{NH}_{4}^{+}$ & Amine & Amine $^{+}$ \\
\hline MAL/1ox/DMA_L & 0.01 & 0.99 & 0.49 & 0.029 & 0.48 & 8.6E-09 & 1 & $3.1 \mathrm{E}-10$ & 1 \\
\hline MAL/10ox/DMA_L & 0.01 & 0.99 & 0.49 & 0.029 & 0.48 & 8.6E-09 & 1 & $3.1 \mathrm{E}-10$ & 1 \\
\hline MAL/100ox/DMA_L & 0.011 & 0.99 & 0.5 & 0.028 & 0.47 & 8.3E-09 & 1 & $3 \mathrm{E}-10$ & 1 \\
\hline MAL/1ox/TMA_L & 0.018 & 0.98 & 0.64 & 0.027 & 0.33 & $6.3 \mathrm{E}-09$ & 1 & 2.3E-09 & 1 \\
\hline MAL/10ox/TMA_L & 0.018 & 0.98 & 0.64 & 0.027 & 0.33 & $6.3 \mathrm{E}-09$ & 1 & 2.3E-09 & 1 \\
\hline MAL/100ox/TMA_L & 0.018 & 0.98 & 0.64 & 0.027 & 0.33 & $6.2 \mathrm{E}-09$ & 1 & 2.3E-09 & 1 \\
\hline MAL/1ox/TMA_T & 0.013 & 0.99 & 0.55 & 0.028 & 0.42 & 7.6E-09 & 1 & $2.8 \mathrm{E}-09$ & 1 \\
\hline MAL/10ox/TMA_T & 0.013 & 0.99 & 0.55 & 0.028 & 0.42 & $7.6 \mathrm{E}-09$ & 1 & $2.8 \mathrm{E}-09$ & 1 \\
\hline MAL/100ox/TMA_T & 0.013 & 0.99 & 0.56 & 0.028 & 0.41 & 7.4E-09 & 1 & 2.7E-09 & 1 \\
\hline OX/1ox/DMA_L & 0.016 & 0.98 & 0.071 & 0.1 & 0.83 & 4.2E-09 & 1 & $1.5 \mathrm{E}-10$ & 1 \\
\hline OX/10ox/DMA_L & 0.01 & 0.99 & 0.028 & 0.066 & 0.91 & 8.6E-09 & 1 & $3.2 \mathrm{E}-10$ & 1 \\
\hline OX/100ox/DMA_L & 0.01 & 0.99 & 0.028 & 0.066 & 0.91 & $8.5 \mathrm{E}-09$ & 1 & $3.1 \mathrm{E}-10$ & 1 \\
\hline OX/1ox/TMA_L & 0.024 & 0.98 & 0.1 & 0.11 & 0.78 & $3.6 \mathrm{E}-09$ & 1 & $1.4 \mathrm{E}-09$ & 1 \\
\hline OX/10ox/TMA_L & 0.025 & 0.97 & 0.11 & 0.12 & 0.77 & $3.1 \mathrm{E}-09$ & 1 & $1.3 \mathrm{E}-09$ & 1 \\
\hline OX/100ox/TMA_L & 0.018 & 0.98 & 0.05 & 0.086 & 0.86 & $6.3 \mathrm{E}-09$ & 1 & 2.3E-09 & 1 \\
\hline OX/1ox/TMA_T & 0.019 & 0.98 & 0.085 & 0.11 & 0.81 & 3.9E-09 & 1 & $1.5 \mathrm{E}-09$ & 1 \\
\hline OX/10ox/TMA_T & 0.013 & 0.99 & 0.035 & 0.074 & 0.89 & 7.6E-09 & 1 & $2.8 \mathrm{E}-09$ & 1 \\
\hline OX/100ox/TMA_T & 0.013 & 0.99 & 0.035 & 0.074 & 0.89 & 7.5E-09 & 1 & $2.8 \mathrm{E}-09$ & 1 \\
\hline MAL_LoVP/1ox/DMA_L & 0.011 & 0.99 & 0.5 & 0.028 & 0.47 & 8.3E-09 & 1 & $3 \mathrm{E}-10$ & 1 \\
\hline MAL_LoVP/10ox/DMA_L & 0.011 & 0.99 & 0.51 & 0.028 & 0.46 & 8.1E-09 & 1 & $2.9 \mathrm{E}-10$ & 1 \\
\hline MAL_LoVP/100ox/DMA_L & 0.011 & 0.99 & 0.56 & 0.028 & 0.41 & $6.9 \mathrm{E}-09$ & 1 & $2.3 \mathrm{E}-10$ & 1 \\
\hline MAL_LoVP/1ox/TMA_L & 0.018 & 0.98 & 0.64 & 0.027 & 0.33 & $6.2 \mathrm{E}-09$ & 1 & $2.3 \mathrm{E}-09$ & 1 \\
\hline
\end{tabular}




\begin{tabular}{llllllllll}
\hline MAL_LoVP/10ox/TMA_L & 0.018 & 0.98 & 0.65 & 0.027 & 0.32 & $6.1 \mathrm{E}-09$ & 1 & $2.3 \mathrm{E}-09$ & 1 \\
MAL_LoVP/100ox/TMA_L & 0.018 & 0.98 & 0.68 & 0.026 & 0.3 & $5.6 \mathrm{E}-09$ & 1 & $1.9 \mathrm{E}-09$ & 1 \\
MAL_LoVP/10x/TMA_T & 0.013 & 0.99 & 0.56 & 0.028 & 0.41 & $7.4 \mathrm{E}-09$ & 1 & $2.7 \mathrm{E}-09$ & 1 \\
MAL_LoVP/10ox/TMA_T & 0.013 & 0.99 & 0.57 & 0.028 & 0.4 & $7.2 \mathrm{E}-09$ & 1 & $2.7 \mathrm{E}-09$ & 1 \\
MAL_LoVP/100ox/TMA_T & 0.013 & 0.99 & 0.61 & 0.027 & 0.37 & $6.3 \mathrm{E}-09$ & 1 & $2.2 \mathrm{E}-09$ & 1 \\
OX_LoVP/10x/DMA_L & 0.01 & 0.99 & 0.028 & 0.066 & 0.91 & $8.5 \mathrm{E}-09$ & 1 & $3.1 \mathrm{E}-10$ & 1 \\
OX_LoVP/10ox/DMA_L & 0.01 & 0.99 & 0.028 & 0.067 & 0.9 & $8.4 \mathrm{E}-09$ & 1 & $3.1 \mathrm{E}-10$ & 1 \\
OX_LoVP/100ox/DMA_L & 0.011 & 0.99 & 0.031 & 0.07 & 0.9 & $7.7 \mathrm{E}-09$ & 1 & $2.8 \mathrm{E}-10$ & 1 \\
OX_LoVP/10x/TMA_L & 0.018 & 0.98 & 0.05 & 0.086 & 0.86 & $6.3 \mathrm{E}-09$ & 1 & $2.3 \mathrm{E}-09$ & 1 \\
OX_LoVP/10ox/TMA_L & 0.018 & 0.98 & 0.05 & 0.086 & 0.86 & $6.2 \mathrm{E}-09$ & 1 & $2.3 \mathrm{E}-09$ & 1 \\
OX_LoVP/100ox/TMA_L & 0.019 & 0.98 & 0.053 & 0.089 & 0.86 & $5.9 \mathrm{E}-09$ & 1 & $2.2 \mathrm{E}-09$ & 1 \\
OX_LoVP/10x/TMA_T & 0.013 & 0.99 & 0.035 & 0.074 & 0.89 & $7.5 \mathrm{E}-09$ & 1 & $2.8 \mathrm{E}-09$ & 1 \\
OX_LoVP/10ox/TMA_T & 0.013 & 0.99 & 0.036 & 0.074 & 0.89 & $7.5 \mathrm{E}-09$ & 1 & $2.8 \mathrm{E}-09$ & 1 \\
OX_LoVP/100ox/TMA_T & 0.013 & 0.99 & 0.039 & 0.077 & 0.88 & $6.9 \mathrm{E}-09$ & 1 & $2.6 \mathrm{E}-09$ & 1 \\
\hline
\end{tabular}


Table S2. Breakdown of the percent dissociations of $\mathrm{H}_{2} \mathrm{SO}_{4}$ to $\mathrm{HSO}_{4}{ }^{-1}$ to $\mathrm{HSO}_{4}{ }^{-1}$ and $\mathrm{SO}_{4}{ }^{-2}$; the organic acid (malonic or oxalic) to its first and second dissociation products; $\mathrm{NH}_{3}$ to $\mathrm{NH}_{4}{ }^{+}$; and the amine (DMA or TMA) to its dissociation product for the model output of May 9, 2013. In MABNAG, sulfuric acid is assumed to dissociate completely

\begin{tabular}{|c|c|c|c|c|c|c|c|c|c|}
\hline Case & $\mathrm{HSO}_{4}^{-1}$ & $\mathrm{SO}_{4}^{-2}$ & Org Acid & $\begin{array}{l}\text { Org. } \\
\text { Acid }^{-1}\end{array}$ & $\begin{array}{l}\text { Org. } \\
\text { Acid }^{-2}\end{array}$ & $\mathrm{NH}_{3}$ & $\mathrm{NH}_{4}^{+}$ & Amine & Amine $^{+}$ \\
\hline MAL/1ox/DMA_L & 0.032 & 0.97 & 0.78 & 0.035 & 0.18 & 3.3E-09 & 1 & $1.4 \mathrm{E}-10$ & 1 \\
\hline MAL/10ox/DMA_L & 0.031 & 0.97 & 0.78 & 0.035 & 0.19 & 3.3E-09 & 1 & $1.4 E-10$ & 1 \\
\hline MAL/100ox/DMA_L & 0.028 & 0.97 & 0.77 & 0.035 & 0.19 & 3.7E-09 & 1 & $1.4 \mathrm{E}-10$ & 1 \\
\hline MAL/1ox/TMA_L & 0.062 & 0.94 & 0.88 & 0.026 & 0.092 & $2.2 \mathrm{E}-09$ & 1 & $1.2 \mathrm{E}-09$ & 1 \\
\hline MAL/10ox/TMA_L & 0.061 & 0.94 & 0.88 & 0.025 & 0.092 & $2.2 \mathrm{E}-09$ & 1 & $1.2 \mathrm{E}-09$ & 1 \\
\hline MAL/100ox/TMA_L & 0.055 & 0.95 & 0.88 & 0.025 & 0.097 & $2.5 \mathrm{E}-09$ & 1 & $1.0 \mathrm{E}-09$ & 1 \\
\hline MAL/1ox/TMA_T & 0.035 & 0.97 & 0.8 & 0.034 & 0.17 & $3.1 \mathrm{E}-09$ & 1 & $1.6 \mathrm{E}-09$ & 1 \\
\hline MAL/10ox/TMA_T & 0.035 & 0.97 & 0.8 & 0.033 & 0.17 & $3.1 \mathrm{E}-09$ & 1 & $1.6 \mathrm{E}-09$ & 1 \\
\hline MAL/100ox/TMA_T & 0.031 & 0.97 & 0.79 & 0.033 & 0.18 & $3.5 \mathrm{E}-09$ & 1 & $1.4 \mathrm{E}-09$ & 1 \\
\hline OX/1ox/DMA_L & 0.032 & 0.97 & 0.13 & 0.16 & 0.71 & 3.2E-09 & 1 & $1.4 \mathrm{E}-10$ & 1 \\
\hline OX/10ox/DMA_L & 0.032 & 0.97 & 0.13 & 0.16 & 0.71 & $3.2 \mathrm{E}-09$ & 1 & $1.4 \mathrm{E}-10$ & 1 \\
\hline OX/100ox/DMA_L & 0.032 & 0.97 & 0.13 & 0.16 & 0.71 & $3.2 \mathrm{E}-09$ & 1 & $1.4 \mathrm{E}-10$ & 1 \\
\hline OX/1ox/TMA_L & 0.062 & 0.94 & 0.24 & 0.19 & 0.57 & 2.2E-09 & 1 & $1.2 \mathrm{E}-09$ & 1 \\
\hline OX/10ox/TMA_L & 0.062 & 0.94 & 0.24 & 0.19 & 0.57 & $2.2 \mathrm{E}-09$ & 1 & $1.2 \mathrm{E}-09$ & 1 \\
\hline OX/100ox/TMA_L & 0.062 & 0.94 & 0.24 & 0.2 & 0.57 & $2.2 \mathrm{E}-09$ & 1 & $1.2 \mathrm{E}-09$ & 1 \\
\hline OX/1ox/TMA_T & 0.035 & 0.97 & 0.14 & 0.17 & 0.69 & $3.0 \mathrm{E}-09$ & 1 & $1.6 \mathrm{E}-09$ & 1 \\
\hline OX/10ox/TMA_T & 0.035 & 0.97 & 0.14 & 0.17 & 0.69 & 3.0E-09 & 1 & $1.6 \mathrm{E}-09$ & 1 \\
\hline OX/100ox/TMA_T & 0.035 & 0.97 & 0.14 & 0.17 & 0.69 & $3.1 \mathrm{E}-09$ & 1 & $1.6 \mathrm{E}-09$ & 1 \\
\hline MAL_LoVP/1ox/DMA_L & 0.03 & 0.97 & 0.78 & 0.034 & 0.19 & $3.5 \mathrm{E}-09$ & 1 & $1.4 \mathrm{E}-10$ & 1 \\
\hline MAL_LoVP/10ox/DMA_L & 0.027 & 0.97 & 0.76 & 0.035 & 0.2 & 4.0E-09 & 1 & $1.4 \mathrm{E}-10$ & 1 \\
\hline MAL_LoVP/100ox/DMA_L & 0.016 & 0.98 & 0.71 & 0.04 & 0.25 & $5.4 \mathrm{E}-09$ & 1 & $1.6 \mathrm{E}-10$ & 1 \\
\hline MAL_LoVP/1ox/TMA_L & 0.057 & 0.94 & 0.88 & 0.025 & 0.094 & $2.4 \mathrm{E}-09$ & 1 & $1.1 \mathrm{E}-09$ & 1 \\
\hline MAL_LoVP/10ox/TMA_L & 0.051 & 0.95 & 0.87 & 0.026 & 0.1 & 2.7E-09 & 1 & $9.9 \mathrm{E}-10$ & 1 \\
\hline MAL_LoVP/100ox/TMA_L & 0.025 & 0.98 & 0.81 & 0.037 & 0.16 & $4.5 \mathrm{E}-09$ & 1 & $9.7 \mathrm{E}-10$ & 1 \\
\hline
\end{tabular}




\begin{tabular}{llllllllll}
\hline MAL_LoVP/10x/TMA_T & 0.033 & 0.97 & 0.8 & 0.033 & 0.17 & $3.3 \mathrm{E}-09$ & 1 & $1.5 \mathrm{E}-09$ & 1 \\
MAL_LoVP/10ox/TMA_T & 0.029 & 0.97 & 0.78 & 0.034 & 0.18 & $3.8 \mathrm{E}-09$ & 1 & $1.3 \mathrm{E}-09$ & 1 \\
MAL_LoVP/100ox/TMA_T & 0.017 & 0.98 & 0.73 & 0.04 & 0.23 & $5.2 \mathrm{E}-09$ & 1 & $1.2 \mathrm{E}-09$ & 1 \\
OX_LoVP/10x/DMA_L & 0.032 & 0.97 & 0.13 & 0.16 & 0.71 & $3.2 \mathrm{E}-09$ & 1 & $1.4 \mathrm{E}-10$ & 1 \\
OX_LoVP/10ox/DMA_L & 0.032 & 0.97 & 0.13 & 0.16 & 0.71 & $3.2 \mathrm{E}-09$ & 1 & $1.4 \mathrm{E}-10$ & 1 \\
OX_LoVP/100ox/DMA_L & 0.029 & 0.97 & 0.12 & 0.16 & 0.71 & $3.3 \mathrm{E}-09$ & 1 & $1.3 \mathrm{E}-10$ & 1 \\
OX_LoVP/10x/TMA_L & 0.062 & 0.94 & 0.24 & 0.2 & 0.57 & $2.2 \mathrm{E}-09$ & 1 & $1.2 \mathrm{E}-09$ & 1 \\
OX_LoVP/10ox/TMA_L & 0.062 & 0.94 & 0.23 & 0.2 & 0.57 & $2.2 \mathrm{E}-09$ & 1 & $1.2 \mathrm{E}-09$ & 1 \\
OX_LoVP/100ox/TMA_L & 0.058 & 0.94 & 0.23 & 0.2 & 0.58 & $2.3 \mathrm{E}-09$ & 1 & $1.1 \mathrm{E}-09$ & 1 \\
OX_LoVP/10x/TMA_T & 0.035 & 0.97 & 0.14 & 0.17 & 0.69 & $3.0 \mathrm{E}-09$ & 1 & $1.6 \mathrm{E}-09$ & 1 \\
OX_LoVP/10ox/TMA_T & 0.035 & 0.97 & 0.14 & 0.17 & 0.69 & $3.1 \mathrm{E}-09$ & 1 & $1.6 \mathrm{E}-09$ & 1 \\
OX_LoVP/100ox/TMA_T & 0.032 & 0.97 & 0.14 & 0.17 & 0.69 & $3.1 \mathrm{E}-09$ & 1 & $1.4 \mathrm{E}-09$ & 1 \\
\hline
\end{tabular}


Table S3. Breakdown of the percent dissociations of $\mathrm{H}_{2} \mathrm{SO}_{4}$ to $\mathrm{HSO}_{4}{ }^{-1}$ to $\mathrm{HSO}_{4}{ }^{-1}$ and $\mathrm{SO}_{4}{ }^{-2}$; the organic acid (malonic or oxalic) to its first and second dissociation products; $\mathrm{NH}_{3}$ to $\mathrm{NH}_{4}{ }^{+}$; and the amine (DMA or TMA) to its dissociation product for the model output of May 11, 2013. In MABNAG, sulfuric acid is assumed to dissociate completely.

\begin{tabular}{|c|c|c|c|c|c|c|c|c|c|}
\hline Case & $\mathrm{HSO}_{4}^{-1}$ & $\mathrm{SO}_{4}^{-2}$ & $\begin{array}{l}\text { Org } \\
\text { Acid }\end{array}$ & $\begin{array}{l}\text { Org. } \\
\text { Acid }^{-1}\end{array}$ & $\begin{array}{l}\text { Org. } \\
\text { Acid }^{-2}\end{array}$ & $\mathrm{NH}_{3}$ & $\mathrm{NH}_{4}^{+}$ & Amine & Amine $^{+}$ \\
\hline MAL/1ox/DMA_L & 0.026 & 0.97 & 0.78 & 0.026 & 0.19 & 3.9E-09 & 1 & $1.8 \mathrm{E}-10$ & 1 \\
\hline MAL/10ox/DMA_L & 0.026 & 0.97 & 0.78 & 0.026 & 0.19 & 3.9E-09 & 1 & $1.8 E-10$ & 1 \\
\hline MAL/100ox/DMA_L & 0.027 & 0.97 & 0.78 & 0.026 & 0.19 & 4.1E-09 & 1 & $1.8 \mathrm{E}-10$ & 1 \\
\hline MAL/1ox/TMA_L & 0.039 & 0.96 & 0.84 & 0.023 & 0.13 & $3.2 \mathrm{E}-09$ & 1 & $2.1 \mathrm{E}-09$ & 1 \\
\hline MAL/10ox/TMA_L & 0.039 & 0.96 & 0.84 & 0.023 & 0.13 & $3.2 \mathrm{E}-09$ & 1 & $2.1 \mathrm{E}-09$ & 1 \\
\hline MAL/100ox/TMA_L & 0.04 & 0.96 & 0.84 & 0.022 & 0.13 & 3.3E-09 & 1 & $2.0 \mathrm{E}-09$ & 1 \\
\hline MAL/1ox/TMA_T & 0.033 & 0.97 & 0.82 & 0.024 & 0.16 & $3.5 \mathrm{E}-09$ & 1 & 2.3E-09 & 1 \\
\hline MAL/10ox/TMA_T & 0.033 & 0.97 & 0.82 & 0.024 & 0.16 & $3.5 \mathrm{E}-09$ & 1 & 2.3E-09 & 1 \\
\hline MAL/100ox/TMA_T & 0.033 & 0.97 & 0.82 & 0.024 & 0.16 & $3.6 \mathrm{E}-09$ & 1 & $2.2 \mathrm{E}-09$ & 1 \\
\hline OX/1ox/DMA_L & 0.033 & 0.97 & 0.15 & 0.14 & 0.71 & 3.3E-09 & 1 & $1.4 \mathrm{E}-10$ & 1 \\
\hline OX/10ox/DMA_L & 0.026 & 0.97 & 0.1 & 0.13 & 0.77 & 3.9E-09 & 1 & $1.8 \mathrm{E}-10$ & 1 \\
\hline OX/100ox/DMA_L & 0.026 & 0.97 & 0.1 & 0.13 & 0.77 & $3.9 \mathrm{E}-09$ & 1 & $1.8 \mathrm{E}-10$ & 1 \\
\hline OX/1ox/TMA_L & 0.046 & 0.95 & 0.19 & 0.16 & 0.66 & $2.8 \mathrm{E}-09$ & 1 & $1.6 \mathrm{E}-09$ & 1 \\
\hline OX/10ox/TMA_L & 0.039 & 0.96 & 0.14 & 0.15 & 0.71 & $3.2 \mathrm{E}-09$ & 1 & $2.2 \mathrm{E}-09$ & 1 \\
\hline OX/100ox/TMA_L & 0.04 & 0.96 & 0.15 & 0.15 & 0.71 & $3.2 \mathrm{E}-09$ & 1 & $2.2 \mathrm{E}-09$ & 1 \\
\hline OX/1ox/TMA_T & 0.041 & 0.96 & 0.17 & 0.15 & 0.68 & 3.0E-09 & 1 & $1.6 \mathrm{E}-09$ & 1 \\
\hline OX/10ox/TMA_T & 0.033 & 0.97 & 0.13 & 0.14 & 0.73 & $3.5 \mathrm{E}-09$ & 1 & 2.3E-09 & 1 \\
\hline OX/100ox/TMA_T & 0.033 & 0.97 & 0.13 & 0.14 & 0.73 & $3.5 \mathrm{E}-09$ & 1 & 2.3E-09 & 1 \\
\hline MAL_LoVP/1ox/DMA_L & 0.026 & 0.97 & 0.78 & 0.026 & 0.19 & 4.0E-09 & 1 & $1.8 \mathrm{E}-10$ & 1 \\
\hline MAL_LoVP/10ox/DMA_L & 0.027 & 0.97 & 0.78 & 0.026 & 0.19 & 4.1E-09 & 1 & $1.7 \mathrm{E}-10$ & 1 \\
\hline MAL_LoVP/100ox/DMA_L & 0.017 & 0.98 & 0.75 & 0.024 & 0.22 & $6.2 \mathrm{E}-09$ & 1 & $1.6 \mathrm{E}-10$ & 1 \\
\hline MAL_LoVP/1ox/TMA_L & 0.04 & 0.96 & 0.84 & 0.022 & 0.13 & 3.3E-09 & 1 & 2.1E-09 & 1 \\
\hline MAL_LoVP/10ox/TMA_L & 0.04 & 0.96 & 0.84 & 0.022 & 0.13 & $3.4 \mathrm{E}-09$ & 1 & $1.9 \mathrm{E}-09$ & 1 \\
\hline MAL_LoVP/100ox/TMA_L & 0.028 & 0.97 & 0.82 & 0.021 & 0.16 & $5.2 \mathrm{E}-09$ & 1 & $1.1 \mathrm{E}-09$ & 1 \\
\hline
\end{tabular}




\begin{tabular}{llllllllll}
\hline MAL_LoVP/1ox/TMA_T & 0.033 & 0.97 & 0.82 & 0.024 & 0.16 & $3.6 \mathrm{E}-09$ & 1 & $2.2 \mathrm{E}-09$ & 1 \\
MAL_LoVP/10ox/TMA_T & 0.034 & 0.97 & 0.82 & 0.024 & 0.16 & $3.7 \mathrm{E}-09$ & 1 & $2.1 \mathrm{E}-09$ & 1 \\
MAL_LoVP/100ox/TMA_T & 0.022 & 0.98 & 0.79 & 0.022 & 0.19 & $5.7 \mathrm{E}-09$ & 1 & $1.1 \mathrm{E}-09$ & 1 \\
OX_LoVP/1ox/DMA_L & 0.026 & 0.97 & 0.1 & 0.13 & 0.77 & $3.9 \mathrm{E}-09$ & 1 & $1.8 \mathrm{E}-10$ & 1 \\
OX_LoVP/10ox/DMA_L & 0.026 & 0.97 & 0.1 & 0.13 & 0.77 & $3.9 \mathrm{E}-09$ & 1 & $1.8 \mathrm{E}-10$ & 1 \\
OX_LoVP/100ox/DMA_L & 0.028 & 0.97 & 0.1 & 0.13 & 0.77 & $3.9 \mathrm{E}-09$ & 1 & $1.8 \mathrm{E}-10$ & 1 \\
OX_LoVP/10x/TMA_L & 0.039 & 0.96 & 0.14 & 0.15 & 0.71 & $3.2 \mathrm{E}-09$ & 1 & $2.2 \mathrm{E}-09$ & 1 \\
OX_LoVP/10ox/TMA_L & 0.04 & 0.96 & 0.15 & 0.15 & 0.71 & $3.2 \mathrm{E}-09$ & 1 & $2.2 \mathrm{E}-09$ & 1 \\
OX_LoVP/100ox/TMA_L & 0.042 & 0.96 & 0.15 & 0.15 & 0.71 & $3.2 \mathrm{E}-09$ & 1 & $2.1 \mathrm{E}-09$ & 1 \\
OX_LoVP/10x/TMA_T & 0.033 & 0.97 & 0.13 & 0.14 & 0.73 & $3.5 \mathrm{E}-09$ & 1 & $2.3 \mathrm{E}-09$ & 1 \\
OX_LoVP/10ox/TMA_T & 0.033 & 0.97 & 0.13 & 0.14 & 0.73 & $3.5 \mathrm{E}-09$ & 1 & $2.3 \mathrm{E}-09$ & 1 \\
OX_LoVP/100ox/TMA_T & 0.035 & 0.97 & 0.13 & 0.14 & 0.73 & $3.4 \mathrm{E}-09$ & 1 & $2.3 \mathrm{E}-09$ & 1 \\
\hline
\end{tabular}

